\title{
The influence of strong perturbations on wall-bounded flows
}

\author{
O.R.H. Buxton ${ }^{1}$, M. Ewenz Rocher ${ }^{1,2}$ and E. Rodríguez-López ${ }^{3}$ \\ 1. Department of Aeronautics, Imperial College London, U.K. \\ 2. Osney Thermo-Fluids Laboratory, University of Oxford, U.K. \\ 3. Aerodynamics and flight Mechanics Research Group, University of Southampton, U.K.
}

\begin{abstract}
Single-point hot-wire measurements are made downstream of a series of spanwise repeating obstacles that are used to generate an artificially thick turbulent boundary layer. The measurements are made in the near field, in which the turbulent boundary layer is beginning to develop from the wall-bounded wakes of the obstacles. The recent paper of Rodríguez-López et al. (2016b) broadly categorised the mechanisms by which canonical turbulent boundary layers eventually develop from wall-bounded wakes into two distinct mechanisms, the wall-driven and wake-driven mechanisms. In the present work we attempt to identify the geometric parameters of tripping arrays that trigger these two mechanisms by examining the spectra of the streamwise velocity fluctuations and the intermittent outer region of the flow. Using a definition reliant upon the magnitude of the velocity fluctuations, an intermittency function is devised that can discriminate between turbulent and non-turbulent flow. These results are presented along with the spectra in order to try to ascertain which aspects of a trip's geometry are more likely to favour the wall-driven or wake-driven mechanism. The geometrical aspects of the trips tested are the aspect ratio, the total blockage and the blockage at the wall. The results indicate that the presence, or not, of perforations is the most significant factor in affecting the flow downstream. The bleed of fluid through the perforations re-energises the mean recirculation and leads to a narrower intermittent region with a more regular turbulent/non-turbulent interface. The near-wall turbulent motions are found to recover quickly downstream of all of the trips with a wall blockage of $50 \%$ but a clear influence of the outer fluctuations, generated by the tip vortices of the trips, is observed in the near-wall region for the high total blockage trips. The trip with $100 \%$ wall-blockage is found to modify the nature of the inner-wall peak of turbulent kinetic energy.
\end{abstract}

\section{Introduction}

We often encounter thick turbulent boundary layers (TBLs), the most prominent example being the atmospheric boundary layer. If we wish to study the aeroelastic loads and fluid-structure interactions on a new skyscraper design experimentally then we require techniques to artificially thicken the TBL generated in wind tunnels. The general necessity for high fidelity high Reynolds number $(R e)$ experiments was assessed, amongst others, by Klewicki (2010). It is desirable to know how these TBLs grow, and how long they develop for, until they exhibit the canonical properties of a natural TBL.

Among the pioneers of artificial BL thickening was Counihan (1969), who used roughness elements and spires to increase the TBL thickness and whose method was later improved upon by Davidson et al. (1996), amongst others. Hunt and Fernholz (1975) provide a good review of such techniques. However, in recent years, with the advent of improvements in experimental facilities and measurement instrumentation, distinctive properties of high Re TBLs have been discovered (e.g. Nickels et al., 2007; Marusic et al., 2010) thereby placing greater emphasis on ensuring that artificially thickened TBLs mimic their natural counterparts.

Klebanoff and Diehl (1952) artificially thickened TBLs using different trips and paid special attention to recover canonical (reflecting the historical state-of-the-art) TBL properties downstream of a transitional/adaptation region. In order to assess how different trip geometries would affect this adaptation region Rodríguez-López et al. (2016a) conducted an extensive study of the properties in the mid- and far-field of artificially generated high Re turbulent boundary layers employing two different families of trips. They reported that the adaptation region was much shorter for the high aspect ratio cylinder-type trips than for the low aspect ratio sawtooth-type trips. To explain this they postulated that there are two distinctive mechanisms dominating the flow features near the obstacles, namely:

- A wall-driven mechanism with a short adaptation region and little interaction between the inner and outer flow regions, present downstream of the cylinder trips. Here the near-wall motions of the incoming, natural TBL are left relatively undisturbed such that the skin friction quickly recovers the same $C_{f}(x)$ trend as a natural turbulent boundary layer. However, the outer part of the TBL characterised by, for 
example the wake parameter $\Pi$, is more sensitive to perturbations (Chauhan et al., 2009) and thus takes longer to recover.

- A wake-driven mechanism with a long adaptation region and strong interaction between the inner and outer flow regions, present downstream of the sawtooth trips. Here, the inner-wall motions were observed to be strongly influenced by the outer motions and the two-point statistics of the turbulent motions revealed that the eddy structure was fundamentally altered relative to the natural TBL, which was not the case for the wall-driven mechanism.

In order to explore the causes of the two different boundary layer thickening mechanisms downstream of the cylinder and sawtooth trips Rodríguez-López et al. (2016b) conducted a particle image velocimetry (PIV) study immediately downstream of the trips. They reported the importance of the dynamics of the turbulent/nonturbulent interface (TNTI) to determining which mechanism, wall-driven or wake-driven, prevailed. When the TNTI was maintained at a relatively constant height above the wall, due to the regularity of the tip vortex shed by the cylinder trips, then the near-wall layer expands by entraining the turbulent fluid of the wakes of the obstacles. Contrastingly, the wake-driven mechanism is characterised by highly energetic motions of the TNTI generated by the vortices shed downstream of the mean recirculation region of the trips. These motions transport fluid across the entire wall-normal extent of the flow and thus disrupt the near-wall motions from their canonical state. Rodríguez-López et al. (2016b) concluded by postulating three distinct geometrical parameters that would govern whether the wall-driven or wake-driven mechanism would prevail. These were the aspect ratio of the trips, the overall blockage ratio and the blockage at the wall. A larger aspect ratio, ceteris paribus, would increase the number of vertical edges, whilst reducing the number of horizontal edges, thereby reducing the significance of spanwise vorticity which was predicted to lead to a more wall-driven flow. Higher blockage ratio would lead to more energetic recirculation downstream of the obstacles, similar to low porosity perforated plates in a free stream (Castro, 1971), and higher wall blockage would increase the disruption to the near-wall motions hence an increase in both of these parameters was predicted to lead to a more wake-driven flow.

Rodríguez-López et al. (2016b) then revisited some existing literature on artificially thickened TBLs to try to view the reported adaptation regions through the prism of either the wall-driven or wake-driven mechanisms. For example, Klebanoff and Diehl (1952) reported that the rods that they tested were rapidly discarded due to their extraordinarily long adaptation region. The high blockage and $100 \%$ wall blockage of such rods would suggest that this long adaptation region can be attributed to the wake-driven mechanism. Another relevant example is the comparison between Kornilov and Boiko (2012), who artificially thickened their TBL using a spanwise distributed array of vertical cylinders, similar to the cylinders trip of Rodríguez-López et al. (2016a,b) and Kornilov and Boiko (2013) who used a grid of horizontal rods. Canonical TBL properties were recovered after an adaptation region when the vertical cylinders were used as a trip but not the horizontal rods (within the domain examined in Kornilov and Boiko (2013)). Consideration of the different trip geometries suggested that this could be due to a transition from the wall-driven to the wake-driven mechanism.

The objectives of this study are therefore to examine the influence of the three different geometrical parameters identified in Rodríguez-López et al. (2016b) as determining the nature of the adaptation region downstream of the trips. In particular, we seek to compare trips in which the aspect ratio, total blockage and wall blockage are varied between one another. We will then examine the spectral content of the velocity fluctuations and the nature of the intermittent region in the outer part of the boundary layer, in which the TNTI resides, to assess whether the geometrical modifications promote wall-driven or wake-driven flows. The structure of this manuscript is as follows. The experimental methods are introduced in $\S 2$ and the results presented in $\S 3$. In particular, we examine the mean and fluctuating velocity profiles in $\S 3.1$, the spectra in $\S 3.2$ and the intermittency of the outer flow in $\S 3.3$ before bringing the results together and discussing their implications in $\S 4$. Finally, the conclusions of the study are drawn in $\S 5$.

\section{Experimental set-up}

\subsection{Wind tunnel and trips}

The experiment was conducted in the Imperial College London closed-circuit 18-inch wind tunnel, which has a $457 \times 457 \times 1500 \mathrm{~mm}$ (Height $\times$ Width $\times$ Length) square test section. This is the same wind tunnel that was used in Rodríguez-López et al. (2016b). The background turbulence intensity at a freestream velocity of $U_{\infty}=10 \mathrm{~ms}^{-1}$ amounts to $0.15 \%$. Whilst this value is relatively high this study is concerned only with the very near field of the flow downstream of the trips, in which the turbulence intensity is high (due to flow separation, for example) meaning that the influence of the freestream turbulence is expected to be negligible. A $1.2 \mathrm{~m}$ long perspex plate, $12 \mathrm{~mm}$ thick, was mounted horizontally at mid-height spanning the whole test section with the leading edge located $300 \mathrm{~mm}$ downstream of the beginning of the test section. An elliptical leading edge with 10:1 aspect ratio ensured smooth laminar flow at the leading edge. A $20 \mathrm{~mm}$ sand paper strip just behind the leading edge thickened the boundary layer to the desired profile. 


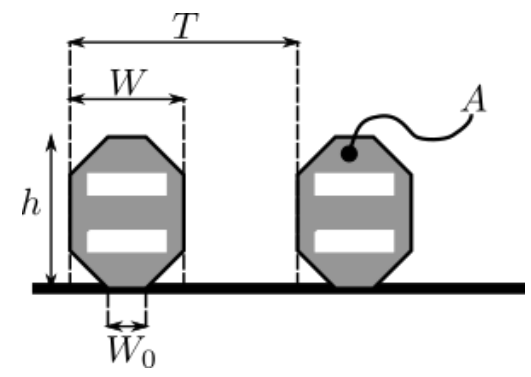

Figure 1: Sketch of a generic trip highlighting the important geometrical features highlighted in table 1

\begin{tabular}{|c|c|c|c|c|}
\hline & base & holes & $A R 2$ & fence \\
\hline$T(\mathrm{~mm})$ & 16 & 16 & 16 & - \\
\hline$W(\mathrm{~mm})$ & 16 & 16 & 8 & - \\
\hline$h(\mathrm{~mm})$ & 16 & 16 & 16 & 16 \\
\hline$d(\mathrm{~mm})$ & - & 6.38 & - & 6.38 \\
\hline$z(\mathrm{~mm})$ & - & 7.5 & - & 7.5 \\
\hline$\sigma(\%)$ & 50 & 25 & 50 & 50 \\
\hline$\sigma_{w}(\%)$ & 50 & 50 & 50 & 100 \\
\hline R & 1 & 1 & 2 & - \\
\hline
\end{tabular}

Table 1: Geometrical parameters for the various trips.

The trips were placed $180 \mathrm{~mm}$ downstream of the leading edge in a machined slot spanning $84 \%$ of the working section width. At this location the incoming boundary layer was estimated to have a thickness $\delta_{I} \approx 3$ mm. A $38.4 \times 2.4 \mathrm{~mm}$ LEGO base plate was glued into the machined slot and the required trips were created in Creo Parametric with the fitting LEGO base. Subsequently, they were 3D printed with photopolymeric materials by a Connex Objet 350 3D Printer. All of the trips tested in this study were of the same thickness of $2 \mathrm{~mm}$. Figure 1 highlights the geometrical dimensions of a generic trip. These are used to define the parameters that Rodríguez-López et al. (2016b) postulated to be important in determining the nature of the transitional region downstream of a generic trip. These are the total blockage $\sigma=A /(T h)$, the blockage at the wall $\sigma_{w}=\sigma(y=0)=W_{0} / T$ (henceforth the wall blockage) and the aspect ratio $R=h / W$. Note that here $A$ is defined as the total frontal area of the trip, i.e. it accounts for the presence (or not) of holes in the trips. The geometry of the tested trips is illustrated and described in table 1 . The trips were designed so that these three parameters could be varied, relative to the base trip, whilst keeping the others constant. The trip names are defined, left to right, as base, holes, AR2 and fence. Whilst a generic trip may have $W_{0} \neq W$ the base, holes and AR2 trips all satisfy $W=W_{0}$ and in all cases $W / T=0.5$ giving a wall blockage of $50 \%$. In contrast the fence trip is solid across the entire spanwise extent of the domain at $y=0$ ( $W$ and $T$ are undefined) yielding a wall blockage of $100 \%$ (of the spanwise extent in which the trips are mounted).

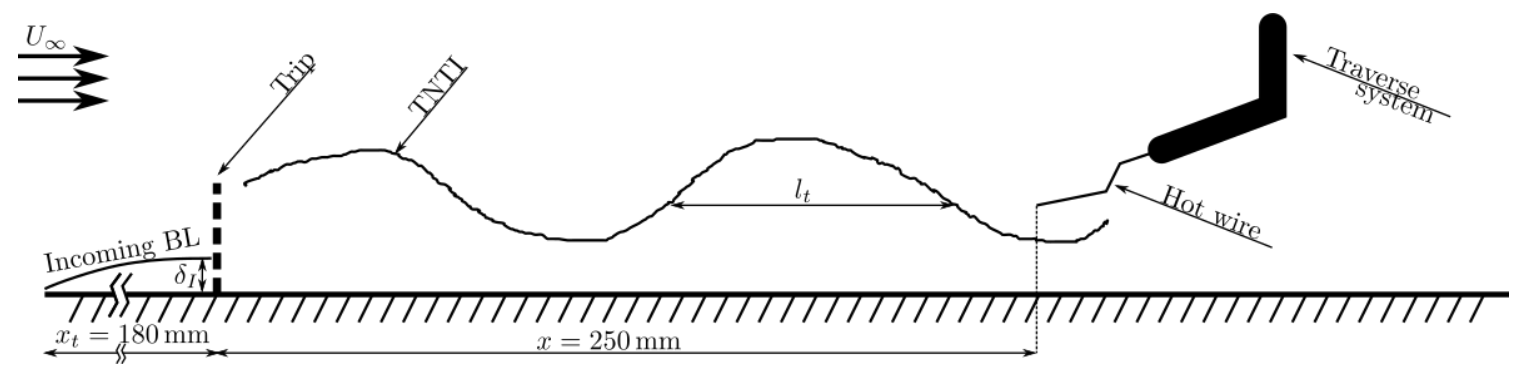

Figure 2: Sketch of the experimental set-up. $l_{t}$ represents the length of a turbulent segment contained between two non-turbulent events. 


\subsection{Hot-wire anemometry}

The hot wire probe was mounted to a probe holder, which in turn was fixed to a rod extending out of the wind tunnel working section connected to a traverse, illustrated in figure 2. The traverse stepping motor was driven by a MSD415 microstep driver allowing positioning of the hot wire with a precision of $0.005 \mathrm{~mm}$. This microstep driver received its signal from a digital I/O connection to the data aqcuisition tool (NI USB 6229 DAQ), which was connected to a computer through USB. This enabled control of the hot wire position through in-house MATLAB routines, which also controlled the wind tunnel motors through PID feedback obtained from a FCO510 Micrometer. Both the DAQ and the microstep driver were powered by an individual power supply. A hot wire was manufactured by soldering a platinum-plated tungsten wire of $d_{w}=5 \mu \mathrm{m}$ diameter to the body of a Dantec 55P01 anemometer. The wire sensor was etched to a sensing length of approximately $l_{w} \approx 1 \mathrm{~mm}$, giving a length to diameter ratio of 200 which is sufficient to ensure that the conductive heat flux is negligible (Comte-Bellot, 1976). The temperature of the hot wire is kept constant at an overheat ratio of 1.8, hence the electrical power required to heat the wire is equal to the convective heat loss rate. One can therefore obtain the fluid velocity from the voltage drop across the sensor. The anemometer is connected to a probe holder which forwards the signal to a Dantec StreamLine (90N10 frame), and subsequently the computer, through a $4 \mathrm{~m}$ long cable. The inner-scaled hot-wire length $l_{w}^{+}=l_{w} u_{\tau} / \nu$ spans values from 16 to 25 for the various trips and no correction is applied for the magnitude of the near-wall turbulence peak. N.B. here $u_{\tau}$ is the friction velocity and its estimation is described in $\S 3.1$ whilst $\nu$ is the kinematic viscosity of the fluid (air).

Measurements were taken in the near field, $250 \mathrm{~mm}$ behind the trip at $U_{\infty}=10 \mathrm{~ms}^{-1}$ freestream velocity. This streamwise measurement location roughly corresponds to the end of the experimental domain of RodríguezLópez et al. (2016b) and the first measurement station in Rodríguez-López et al. (2016a). To account for spanwise variations, each experiment was conducted twice: once behind the obstacle and once behind the gap. With the help of a digital microscope, silver tape and proper illumination the probe was placed near the wall. It was moved towards the wall in steps as small as $0.02 \mathrm{~mm}$ until the hot wire and its mirror image (almost) touched. The measurement points were spaced approximately logarithmically, since the flow characteristics change across much smaller length scales in the inner boundary layer than in the freestream.

The data was sampled at an acquisition frequency of $100 \mathrm{kHz}$ for a duration of $30 \mathrm{~s}$ at each measurement station. A conservative estimate of the shedding frequency of the base trips is $S t \cdot U_{\infty} / h \approx 60 \mathrm{~Hz}$, in which $S t=\mathcal{O}(0.1)$ is the shedding Strouhal number. In this case $30 \mathrm{~s}$ corresponds to $\approx 1,800$ shedding cycles which is sufficient to ensure convergence of both first and second order statistical moments. Note that not only is this a conservative estimate for the shedding frequency of the base trips but that the base trips are also the most conservative case in that we expect the characteristic geometry for the other trips to be smaller, either through thinner obstacles or holes "breaking up" the turbulent structures, thereby yielding a higher shedding frequency and hence sampling over a greater number of shedding cycles. After each measurement, the hot wire was calibrated at the last wall-normal location, in the freestream. The wind tunnel was run at 15 different speeds ranging from $1.5 \mathrm{~ms}^{-1}$ to $15 \mathrm{~ms}^{-1}$ and the mean velocity read from the micro-manometer was matched with the mean voltage read from the hot wire. For this purpose, the velocity read came from a second pitot tube (Pitot 2), which was placed just above the hot wire, instead of the pitot tube mounted upstream of the plate (Pitot 1) used to control the wind tunnel speed. However, Pitot 2 had to be removed for near-wall measurements meaning that the velocity was read from Pitot 1 during the experiment with a correction factor of 0.94 to account for the contraction in the working section. The correction factor had to be adjusted slightly to the room temperature, but was otherwise constant since the flow was incompressible at the testing speeds. If the temperature change across one set of measurements exceeded $0.5 \mathrm{~K}$, the experiment was repeated.

The calibration was performed according to King's law, which in it simplest form may be written as

$$
E^{2}=A+B U^{c} .
$$

Each experiment, with subsequent calibration, yielded an array of voltage $E$ versus velocity $U$ curves and hence coefficients $A, B$ and $c$. Due to noise related issues in which the electronics picked up high-frequency noise emanating from the wind tunnel motor circuit, the resulting velocity signal was low-pass filtered at $6 \mathrm{kHz}$, which was adequate for the purposes of this study.

\section{Results}

\subsection{Mean and root-mean-square profiles}

In this manuscript we decompose the streamwise component of velocity (the only component measured) according to the Reynolds decomposition, $U=u+u^{\prime}$. Figure 3 shows the mean velocity profiles generated both downstream of the obstacles and downstream of the gaps for the various trips at the measurement location of $250 \mathrm{~mm}(=15.6 h)$ downstream. The mean velocity profiles, $u^{+}\left(y^{+}\right)$, are all normalised with mean wall shear 


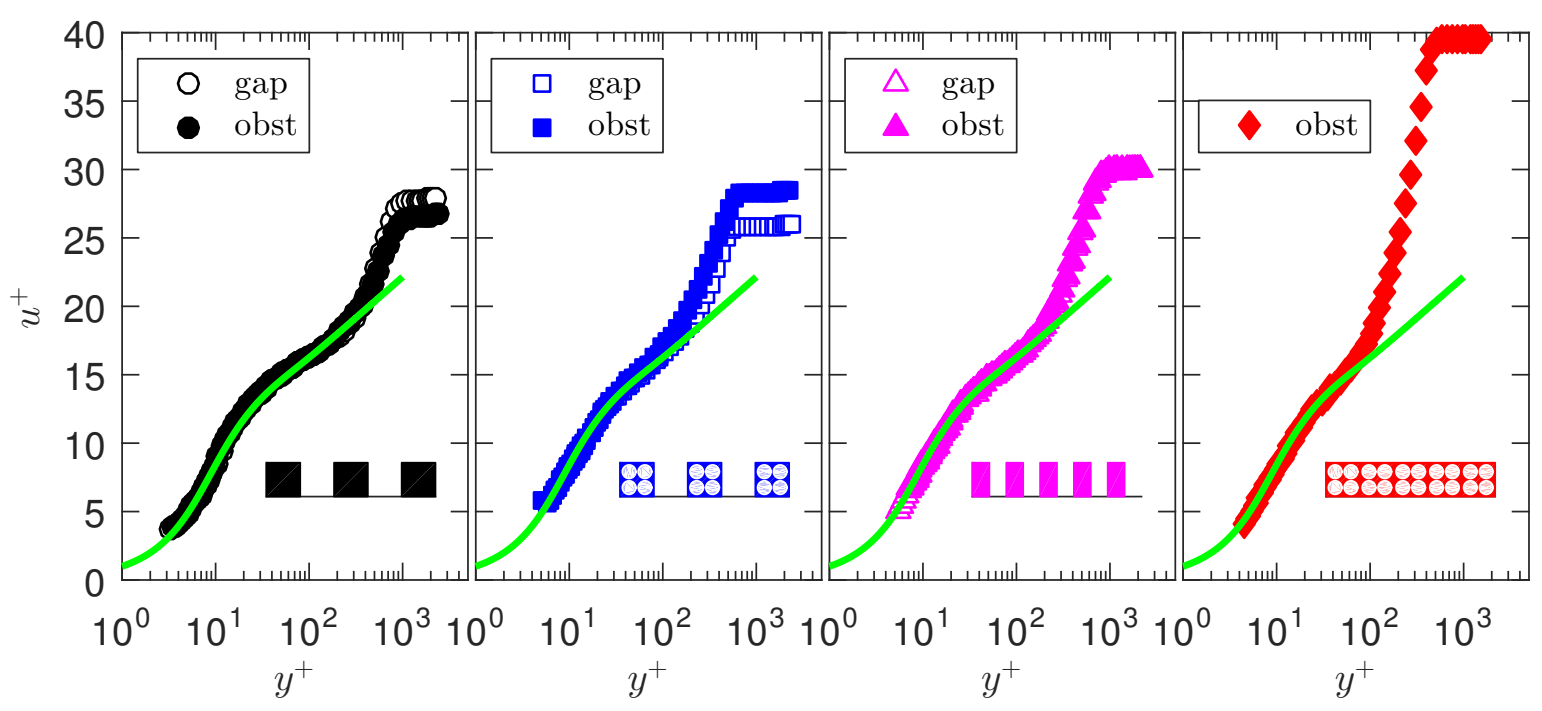

Figure 3: Inner-normalised mean velocity $\left(u^{+}\right)$profile for the various trips and spanwise locations. The solid green line shows the Musker (1979) profile which links the linear $\left(u^{+}=y^{+}\right)$and $\operatorname{logarithmic}\left(u^{+}=\log \left(y^{+}\right) / \kappa+\right.$ $B)$ regions with constants $\kappa=0.38$ and $B=4.17$.

stress, $\tau_{w}$, through the skin friction velocity, $u_{\tau}=\sqrt{\tau_{w} / \rho}$, such that $u^{+}=u / u_{\tau}$ and $y^{+}=y / \delta_{\nu}$ in which $\delta_{\nu}=\nu / u_{\tau}$ is the viscous wall unit. Here all symbols have their usual meaning such that $\rho$ is the fluid (air) density and $y$ is the wall-normal coordinate.

The mean wall shear stress is directly computed from the raw, mean velocity profiles themselves using the methodology of Rodríguez-López et al. (2015). The reader is directed to Rodríguez-López et al. (2015) for a full exposition of the method itself, but briefly it relies upon solving a constrained optimisation problem for $u_{\tau}, \Delta y$ the wall-normal offset of the first measurement location, $\kappa$ the von Kármán constant, $\Pi$ the wake parameter and $\delta$ the boundary-layer thickness. The optimisation is performed such that an error relative to an assumed, canonical form of the mean velocity profile for a zero pressure gradient turbulent boundary layer is minimised. The canonical profile that is chosen is that described in Musker (1979), which links the linear sublayer $u^{+}=y^{+}$to the logarithmic layer $u^{+}=\log \left(y^{+}\right) / \kappa+B$, with the additional "bump" as described in Chauhan et al. (2009) switched on. Here $B$ is simply a constant which has an empirical dependence on $\kappa$ as detailed in Nagib and Chauhan (2008). The outer part of the canonical profile takes after the description of Coles (1956) with the wake function mathematically described as in Chauhan et al. (2009). The complete form of the canonical profile is described in its entirety in Rodríguez-López et al. (2015). This optimisation procedure has been validated against experimental measurements of the wall shear stress and has proven to be robust in determining the skin friction for flows with pressure gradients (Rodríguez-López et al., 2015) and freestream turbulence (Esteban et al., 2017). Further, the method was shown to be robust in determining the wall shear stress in heavily disrupted flows through validation against wall-mounted hot-wire measurements downstream of a variety of wall mounted grids, conceptually similar to the fences trip in the current work, in Rodríguez-López et al. (2017b). Nevertheless, the flows that we consider in the present work are strongly perturbed and thus are not expected to be adequately described by the canonical profile. Nevertheless, Rodríguez-López et al. (2016a) showed that the mean profile of the inner region of the boundary layer (loosely defined as $y^{+} \lesssim 100$ ) does in fact behave in a canonical fashion even for the most draconian tripping condition tested. As such, an uneven weighting is imposed on the data points (using the inverse of $u$ ) biasing the merit function of the optimisation to the inner region of the various boundary layers. Additionally, little merit was placed on the outer parameters $\Pi$ and $\delta$ of the optimisation as the objective was only to compute the wall shear stress $\tau_{w}$ (and adjust for any wall-normal offset that entered through uncertainty in the digital microscope-based procedure). Whilst $\kappa$ was left to float in the optimisation no emphasis is placed on the values returned other than to check that they were within the accepted range presented within the literature (e.g. Marusic et al., 2013; Bailey et al., 2014). All of the mean profiles of figure 3 show the canonical, inner mean velocity profile of Musker (1979) computed assuming fixed logarithmic layer constants of $\kappa=0.38$ and $B=4.17$, superimposed as a green line.

It can be seen that the canonical description of the inner wall mean velocity profile fits that for the perturbed boundary layers extremely well whilst the outer layer, as expected, diverges for all four perturbed flows. For the base, holes and AR2 trips, in which there is a spanwise repeating pattern to the blockage, it can also be seen that there is a great deal of overlap between the profiles extracted from downstream of the obstacles and the profiles extracted from downstream of the gaps. This spanwise homogeneity, at least from behind the obstacles to the gaps (which are the two extremes), for all three spanwise repeating trips reflects the findings 
in Rodríguez-López et al. (2016b) for the trips that they tested. It is interesting to note that the normalised freestream velocity, $U_{\infty}^{+}=u^{+}\left(y^{+} \rightarrow \infty\right)$, is higher downstream of the obstacles for the holes trip which is the opposite behaviour than for the base trip. Higher $U_{\infty}^{+}$(for a fixed $U_{\infty}$ ) is indicative of a reduced wall shear stress which suggests that the interaction of the jets emanating from the holes within the trip interact with the near-wall layer to reduce the wall shear stress. This finding is reinforced with the observation of higher $U_{\infty}^{+}$ downstream of the fence trip, the only other trip containing holes. However, the fence trip has a value of $U_{\infty}^{+}$ that is significantly higher than any of the other trips and the difference between its value and that of any of the other trips is significantly greater than the difference between the value downstream of the holes obstacle and the gap. The fence trip is the only one with a wall blockage of $100 \%$ (the others all have $50 \%$ ) and thus it seems (at this measurement station) that high wall blockage leads to a noticeable reduction in the wall shear stress.

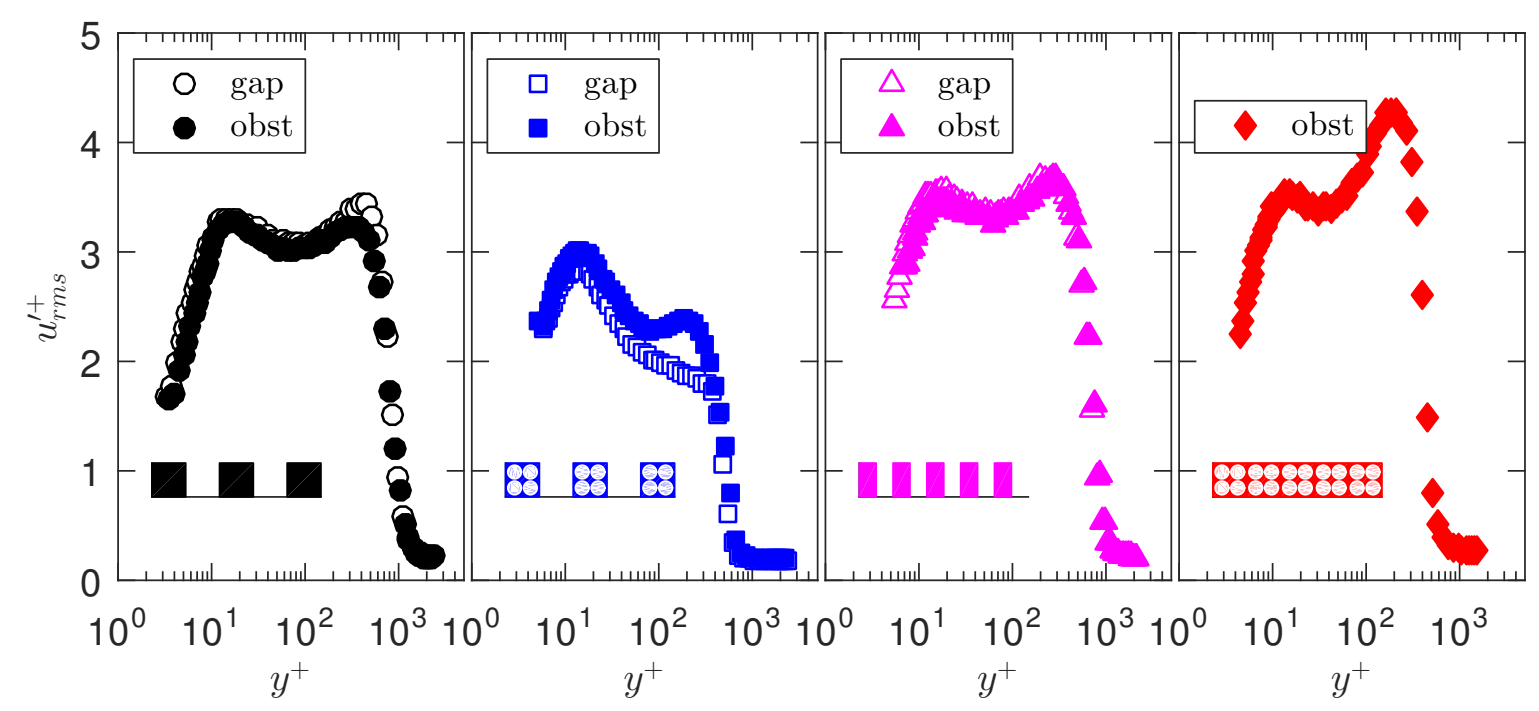

Figure 4: Inner-normalised root-mean-square $\left(u_{r m s}^{\prime+}\right)$ profile for the various trips and spanwise locations.

Figure 4 presents the root-mean-square velocity fluctuation profiles, $u_{r m s}^{\prime+}\left(y^{+}\right)$, for all four different trips. All four trips, with the exception of the flow downstream of the gap for the holes trip, display both an inner and an outer peak of velocity fluctuations intensity viz. turbulent kinetic energy. The inner peak for all four flows is located at $y^{+} \approx 15$, as widely reported for undisturbed zero pressure gradient turbulent boundary layers (e.g. Hutchins and Marusic, 2007), which is associated with the presence of streaks (Kline et al., 1967). This inner-wall peak of turbulence intensity is linked to the self-sustaining mechanisms for turbulence generation in the near-wall region and develops in a much shorter streamwise distance from the trips than other turbulent properties of the boundary layer, such as the two-point statistics or outer motions (Rodríguez-López et al., 2016a). Its existence has also been shown to be robust to the presence of strong freestream turbulence (Dogan et al., 2016). Figure 4 thus provides further evidence for the robustness of this inner-wall peak to perturbations to turbulent boundary layers, regardless of the specifics of the perturbations caused (i.e. different trip geometries). Whilst the values of $\left(u_{r m s}^{\prime+}\right)_{\max }$ differ from trip to trip their wall-normal location remains constant and little difference is noticed between the inner peaks downstream of the obstacles and the gaps. This is of particular interest in the base and holes cases since figure 3 revealed that $U_{\infty}^{+}$(hence $u_{\tau}$ ) was actually slightly different downstream of the obstacles and the gaps. Possible reasons for the differing values of $\left(u_{r m s}^{\prime+}\right)_{\max }$ from trip to trip are the differing wall shear stress and possible interactions between the different energy levels in the outer regions, due to the differing natures of the separated shear layers of the various flows discussed below.

All four profiles of $u_{r m s}^{\prime+}\left(y^{+}\right)$also show an outer peak, which is associated to the shear layer that develops between the free stream and the wakes of the trips. It can be seen that the magnitude of this peak is comparable to, if not slightly larger than, that of the inner peak for the base and AR2 trips, and that it is similar when comparing the flow downstream of the gaps and the obstacles. The magnitude of the outer peak is slightly higher for the AR2 trip than the base trip. This could be explained by the presence of a stronger tip vortex being shed from a higher aspect ratio trip (for an identical total blockage) indicating that higher $R$ gives rise to a more energetic outer peak. In contrast, the magnitude of $\left(u_{r m s}^{\prime+}\right)_{\max }$ for the holes trip is smaller than that for the inner peak and there is a significant discrepancy between the profiles downstream of the obstacles and the gaps, to the extent that no outer peak is evident downstream of the gaps. We thus conclude that the higher blockage trips (base and AR2) have far more two-dimensional wakes than the lower blockage holes. Further, the effects of perforations in the holes trip are to re-energise the recirculation region downstream of the trips and therefore reduce the strength of the shear layer that develops as the flow separates at the tip of the trips 
relative to the solid trips. The final observation is that the strength of the shear layer is particularly strong for the fence trip, significantly higher fluctuations are observed than in the inner peak. This can be attributed to the lack of any gaps in the trip $(100 \%$ blockage at $y=h)$ leading to a strong shear layer forming as the flow separates from the top edge of the trip, similar to the flows produced by wall mounted fences reported in Rodríguez-López et al. (2017a).

\section{$3.2 \quad$ Spectra}

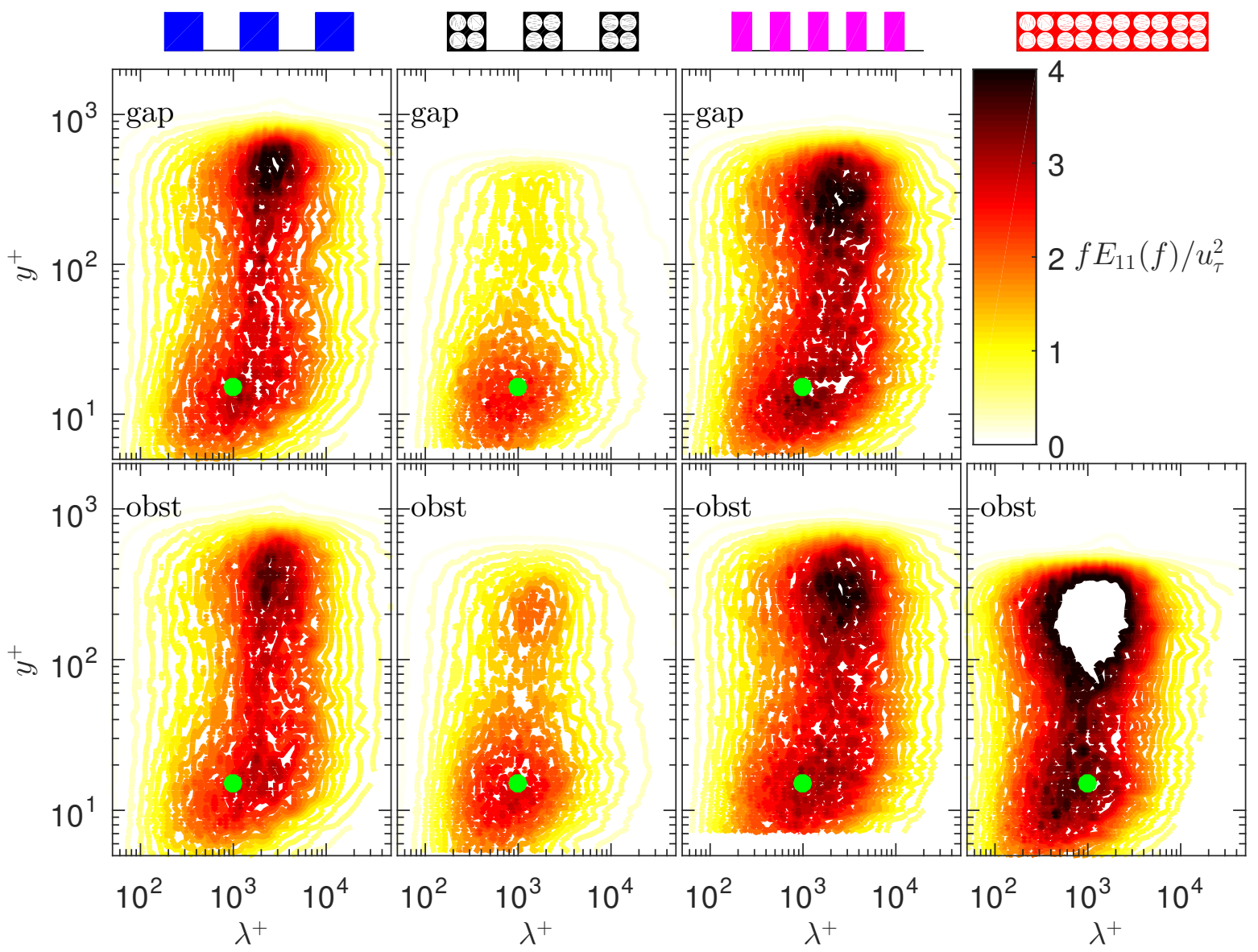

Figure 5: Contour maps of inner-normalised pre-multiplied spectra $\left(f E_{11} / u_{\tau}^{2}\right)$ for the various trips and spanwise locations. The solid green dot highlights the location of $y^{+}=15, \lambda^{+}=1000$. Note that the white portion of the contour map in the lower right figure is simply a saturation of the contour colour map.

The spectral content of the velocity fluctuations is illustrated in figure 5. Contours are plotted of the pre-multiplied one-dimensional energy spectrum, $f E_{11}(f)$, normalised by the inner quantity $u_{\tau}^{2}$ against inner normalised length scale $\lambda^{+}=\lambda / \delta_{\nu}$. Here, length scale $\lambda$ was formed by converting frequency $f$ into space assuming Taylor's hypothesis (Taylor, 1938) in which the advection velocity is equal to the local mean velocity, i.e. $u_{a}(y)=u(y)$, such that $\lambda=u_{a} / f$. Note that one can recreate figure 4 from figure 5 simply by integrating $E_{11}(y)$ across all $\lambda^{+}$(i.e. $f$ ). A green dot is marked onto all the spectral maps of figure 5 at $y^{+}=15, \lambda^{+}=1,000$ which has frequently been reported to be the length-scale associated to the inner wall peak for high Reynolds number wall-bounded turbulent flows (e.g. Marusic et al., 2010).

It can be seen that for the base, holes and $A R 2$ trips (downstream of the obstacles) there are two distinct peaks (as expected from figure 4) but that these two peaks occur at different length scales. In all three cases the inner peak is located close to $\lambda^{+} \approx 1,000$, indicating that this inner peak is due to "canonical" near-wall turbulence production processes. This is in agreement with the observations made for the weak/ "optimal" trips in the study of Sanmiguel Vila et al. (2017). Where an outer peak exists (it does not exist for the holes trip downstream of the gap) it is clear that the preferential length scale is shifted to a larger value of $\lambda^{+}$than the inner peak $\left(\lambda^{+} \approx 2,500\right.$ for the solid, base and AR2, trips and $\lambda^{+} \approx 1,500$ for the holes trips). There is, however, a broader inner peak for the solid trips, extending to larger length scales that are comparable to those for the outer peak, whereas there is a more distinct separation in scales for the holes trip. Contrastingly, the inner peak for the fence case is located at $\lambda^{+} \approx 700$ which is, uniquely in this data set, shifted to a smaller length scale than the expected value of $\lambda^{+} \approx 1,000$. Note that this shifted length scale for the inner turbulence peak 
is accompanied with a higher normalised freestream velocity, $U_{\infty}^{+}$, hence lower wall shear stress as discussed in §3.1. The outer peak for the fence trip has a significantly larger magnitude than any of the other trips due to the energetic shedding triggered by the $100 \%$ blockage at $y=h$. Nevertheless, it appears at a length scale that is similar to that for the holes trips at $\lambda^{+} \approx 1,500$. The qualitative shape of the spectral contour map of the fence trip is similar to that for the strong trips tested by Sanmiguel Vila et al. (2017).

The fact that the outer peak seems to be generated by the presence of a shear layer between the trips and the freestream suggests that the frequency at which it appears may depend on the streamwise distance from the trips. In fact, if we assume that the typical eddies in the shear layer scale with its thickness, this is expected to grow with $x$. Hence, special care should be taken when assessing the frequency content of the outer peak. However, it is clear that at this streamwise location, no matter at which frequency the outer peaks appear, there is a clear penetration of that frequency into the near-wall region. Further, it seems that that this contribution may seem slightly larger in the base case as a consequence of an enhanced outer-inner interaction.

\subsection{Intermittency region}

Rodríguez-López et al. (2016b) identified the behaviour of the turbulent/non-turbulent interface (TNTI) and the intermittent, outer region of the flow, in which a probe may record either turbulent or non-turbulent fluid according to the instantaneous location of the TNTI, as being an important signature of both the wake-driven and the wall-driven mechanisms. Defining the intermittent region relies upon identifying whether the probe is measuring turbulent or non-turbulent fluid. This is often done by setting a threshold on the enstrophy (vorticity magnitude) and defining turbulent fluid as having an enstrophy in excess of a threshold value (e.g. Bisset et al., 2002). However, this is not possible from single probe hot-wire measurements so we instead use a velocity fluctuation intensity, $\hat{k}$, in place of the enstrophy. This is a modified version of the interface detection parameter proposed by Chauhan et al. (2014) and may be expressed as

$$
\hat{k}=\frac{100}{U_{\infty}^{2}} \frac{1}{3} \sum_{m=-1}^{1}\left(u_{l+m}^{\prime}-U_{\infty}\right)^{2}
$$

in which the index $l$ is an arbitrary position within the time series of data and summation over index $m$ indicates a mean over three consecutive measurements in the time series.

We may subsequently define turbulent fluid as that for which $\hat{k}$ exceeds a chosen threshold value and, accordingly, non-turbulent fluid as that for which $\hat{k}$ is below the threshold value. Having defined turbulent and non-turbulent fluid we may define an intermittency parameter, $\gamma(y)$, which denotes the proportion of time that the hot-wire probe records turbulent fluid. Hence close to the wall, where the flow is always turbulent, $\gamma(y \rightarrow 0)=1$ whilst far away from the wall, in the free stream, $\gamma(y \rightarrow \infty)=0$. This definition can be compared to the estimation proposed by Klebanoff (1955), which is based on the wall-normal profile of the kurtosis of the velocity fluctuations,

$$
\gamma_{K}=\frac{3 u_{r m s}^{\prime 2}}{\overline{u^{\prime 4}}} .
$$

A region thus exists in which $0<\gamma(y)<1$ in which the probe will instantaneously record turbulent or nonturbulent fluid which we shall refer to as the intermittency region. By definition the TNTI must reside in this intermittent region, and its extent is indicative of the range of motion of the TNTI. Rodríguez-López et al. (2016b) identified the wake-driven mechanism as being driven by large excursions of the TNTI in the wall-normal direction generated by an unsteady, and energetic, separation downstream of the boundary layer trips, which would result in a thick intermittent region. Contrastingly, when the intermittent region is thinner the TNTI is much less tortuous and remains at a more constant wall-normal height which was observed concomitantly to the wall-driven mechanism.

The intermittency function, $\gamma$, is dependent on identifying turbulent fluid for which $\hat{k}>\hat{k}_{\text {thresh. }}$ and is thus reliant on defining a threshold value which must be carefully chosen. Evidently the threshold value should be set as low as possible in order to correctly identify non-turbulent fluid however, due to freestream turbulence (described in §2.1) the value cannot be set too low and must accurately capture $\gamma=0$ in the freestream. Further, it is desirable to choose a threshold value that does not affect the quantitative results that are obtained, i.e. the thickness of the intermittent region. A sensitivity analysis was thus performed to see the lowest value of $\hat{k}$ that was able to accurately distinguish between turbulent and non-turbulent fluid whilst ensuring that the results were insensitive to small changes in the threshold value chosen.

Figure 6 shows the intermittency profiles, $\gamma(y)$, for the base trips, computed using various threshold values $0.05 \leq \hat{k}_{\text {thresh }} \leq 0.4$ as outlined in the left-hand inset. Klebanoff (1955) showed that a satisfactory fit through his intermittency data could be achieved using an error function

$$
\gamma=\frac{1}{2}\left[1-\operatorname{erf}\left(\frac{y / \delta_{99}-\delta_{\gamma} / \delta_{99}}{s}\right)\right]
$$



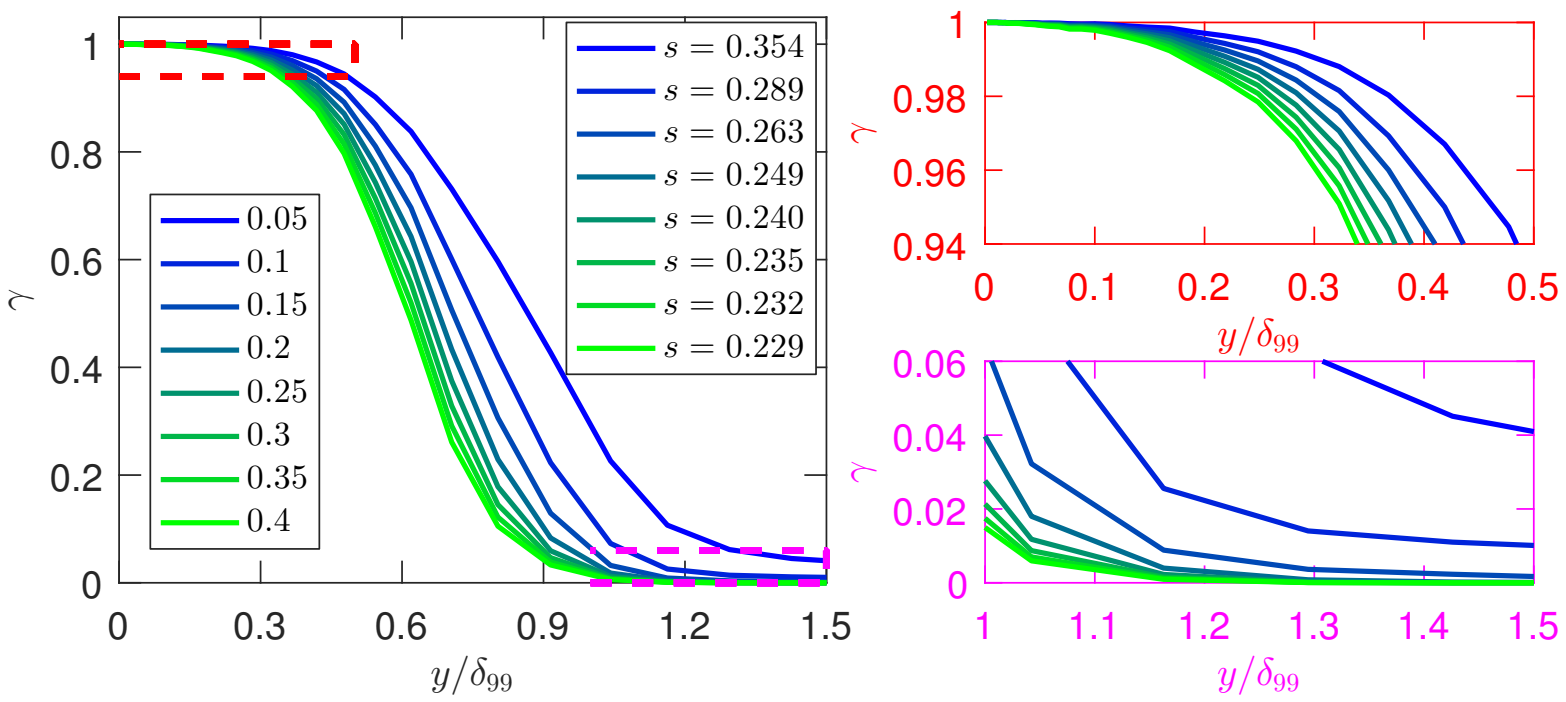

Figure 6: Intermittency profile $\gamma(y)$, defining the proportion of time for which the probe records turbulent fluid, for the base case behind an obstacle. $\gamma(y)$ is computed for a variety of threshold values $\hat{k}_{t h r e s h}$. listed in the bottom left inset. The two plots in the right column highlight the behaviour at high and low values of $\gamma$. The value of $s$ obtained by fitting (4) to the data is also shown.

in which $\delta_{99}$ is the boundary-layer thickness (defined as the wall normal location at which $u=0.99 U_{\infty}$ ). Both $\delta_{\gamma}$ and $s$ are fitting parameters to map the intermittency profiles to (4). $\delta_{\gamma}$ can be understood as a measurement of the local boundary-layer thickness and is approximately equivalent to $0.76 \delta_{99}$ for a zero pressure-gradient TBL as proposed by Klebanoff (1955). Moreover, showing that $\gamma$ follows an error function described by equation (4) is equivalent to showing that the wall-normal position of the TNTI follows a Gaussian process of standard deviation $s$. Hence the thickness of the intermittent region (understood as the region of the flow where $0<\gamma<1$ ) is directly proportional to $s$ for a given confidence interval. We can therefore use $s$ as a quantitative measurement of the intermittent-region thickness. The figure additionally shows the values of $s$ obtained by fitting (4) to the intermittency profiles defined according to the various thresholds, $\hat{k}_{t h r e s h}$. It can readily be seen that at higher threshold values the intermittent region becomes thinner with correspondingly smaller values of $s$. Henceforth, all subsequent results are derived from taking $\hat{k}_{\text {thresh. }}=0.15$ which was shown to be optimum according to the requirements laid out above. It should be noted that this threshold value corresponds to the freestream turbulence intensity level and is therefore, in a sense, the minimum value of the threshold that could be chosen to ensure $\gamma=0$ as $y \rightarrow \infty$.

\begin{tabular}{|c|c|c|c|c|c|c|c|c|}
\hline & \multicolumn{2}{|c|}{ base } & \multicolumn{2}{|c|}{ holes } & \multicolumn{2}{|c|}{$A R 2$} & \multicolumn{2}{|c|}{ fence } \\
\hline Span & gap & obst & gap & obst & gap & obst & gap & obst \\
\hline$\delta_{\gamma} / \delta_{99}$ & 0.778 & 0.709 & 0.805 & 0.789 & 0.769 & 0.769 & - & 0.839 \\
\hline$s$ & 0.253 & 0.263 & 0.149 & 0.166 & 0.226 & 0.229 & - & 0.158 \\
\hline$\delta_{99}(\mathrm{~mm})$ & 42.5 & 45.6 & 22.3 & 27.1 & 37.5 & 37.4 & - & 30.1 \\
\hline$\theta(\mathrm{mm})$ & 5.96 & 5.19 & 3.30 & 3.70 & 5.19 & 5.28 & - & 4.89 \\
\hline
\end{tabular}

Table 2: Fitting parameters $\delta_{\gamma} / \delta_{99}$ and $s$ of the Gaussian intermittency fit (equation (4)) for various trips and spanwise locations. The value of the boundary-layer thickness is also shown in the last two rows.

Figure 7 presents the intermittency functions downstream of both the gaps and the obstacles (where relevant) for all four different trips. The filled symbols are the values of $\gamma$ that are directly measured, the crosses are the values of the Klebanoff (1955) intermittency function of (3) and the lines represent the fitting of (4) to $\gamma(y)$. The values of $\delta_{\gamma} / \delta_{99}$ and $s$ obtained by fitting (4) to the intermittency profiles from the various trips are presented in table 2. Broadly, it can be seen that the different trips can be split into two groups, those with (holes, fence) and without (base, AR2) perforations. Those trips that are perforated exhibit a steeper gradient $\mathrm{d} \gamma / \mathrm{d} y$ in the intermittent region and appreciably smaller values of $s$ than those without perforations. It has been previously shown that downstream of a perforated plate there is a much smaller peak in the spectrum of the energy content of the velocity fluctuations than for an equivalent solid plate (de Bray, 1957; Steiros et al., 


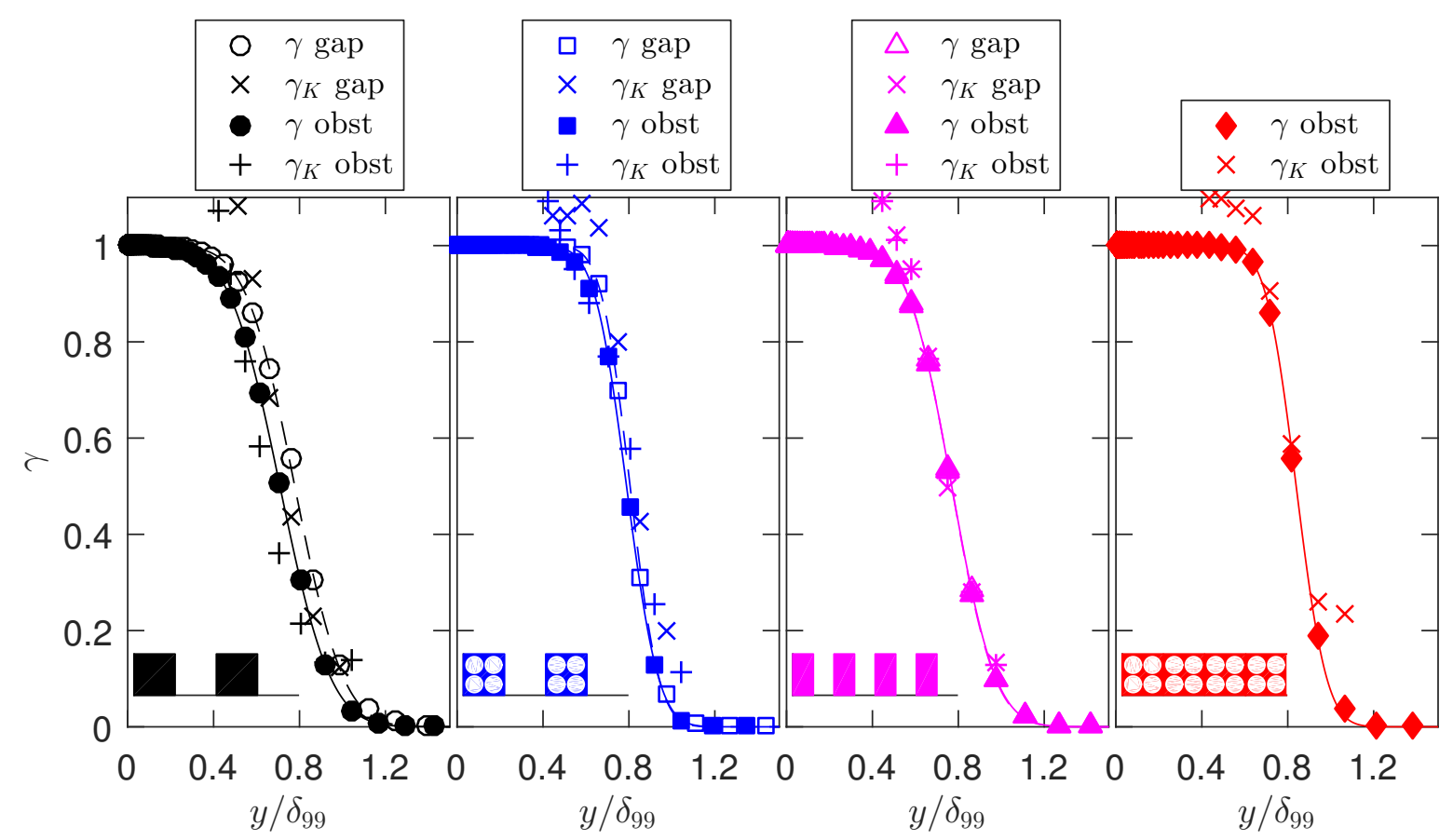

Figure 7: Wall-normal intermittency profiles for various trips and spanwise locations. Solid and dashed lines represent the Gaussian fitting of (4) to the data behind an obstacle or a gap respectively.

2017), indicating a much less energetic shedding mechanism. The large tortuosity of the TNTI, hence thickness of the intermittent region, was attributed to energetic shedding mechanisms by Rodríguez-López et al. (2016b). Figure 7 and table 2 indicate that perforated trips, due to bleed through of high pressure fluid upstream of the trips into the recirculation region downstream, lead to a de-energised shedding and subsequently narrower intermittent region with a high wall-normal gradient. Note that this is primarily reflected in the parameter $s$, whereas $\delta_{\gamma}$ is actually marginally larger for the porous trips than the solid trips. This de-energised shedding downstream of the perforated trips is also associated with a weaker vortex street/shear layer (Castro, 1971) and indeed figure 4 shows a significantly de-energised outer peak downstream of the obstacles for the holes trip relative to the base and $A R 2$ trips. Note that the extremely high magnitude outer peak for the fence trip is due to the $100 \%$ blockage at $y=h$ despite being perforated.

The intermittency function, $\gamma$, tells us about the proportion of time that the probe measures turbulent fluid but it does not reveal anything about the (ir)regularity of the transitions from turbulent to non-turbulent flow. Figure 2 defines length $l_{t}$ as the length of a continuous stream of turbulent fluid contained between two nonturbulent events. This length is computed from the time series of $\hat{k}$ with the time between instances at which $\hat{k}$ drops below threshold $\hat{k}_{\text {thresh }}$. converted into length $l_{t}$ using Taylor's hypothesis with the local mean assumed to be the correct advection velocity. The statistics of $l_{t}$ are collected and presented in figure 8 , which shows probability density functions $(P D F \mathrm{~s})$ of $l_{t}$ for all four trips at three different wall-normal heights accounting for high, medium and low values of $\gamma$ (increasing distance, $y$, away from the wall) which are broadly comparable across the trips. It can be seen that when $\gamma \approx 0.5$ there is very little difference between the different trips; these differences are only apparent in the wall-normal locations with a low $(\gamma \approx 0.2)$ and high $(\gamma \approx 0.8)$ value of the intermittency function. The fence trip produces significantly longer turbulent segments closer to the wall $(\gamma \approx 0.8)$ than the other trips. The $P D F$ is also flat, until it falls away sharply, indicating that there is a broad distribution of length scales present in the flow up until quite close to the minimum wall-normal position of the TNTI. Contrastingly, further away from the wall $(\gamma \approx 0.2)$ it can be seen that the turbulent segments are shorter than for the other trips and the $P D F$ falls away rapidly after about $l_{t} \approx h$. This rapid fall off is also observed for the holes trips, whereas it is significantly more shallow for the AR2 trips than the others

\section{Discussion}

Before discussing the influence of the various parameters case by case, let us consider the variation of the boundary-layer thickness for the various trips since the primary objective of these trips is to generate a thicker TBL. The boundary-layer thickness for the various cases is shown in table 2 where $\theta=\int_{0}^{\infty} u / U_{\infty}\left(1-u / U_{\infty}\right) \mathrm{d} y$ is the momentum thickness. Despite the uncertainties associated with $\delta_{99}$ and $\theta$ the overall trends are maintained for both variables hence the following discussion is valid regardless of which boundary-layer thickness is 


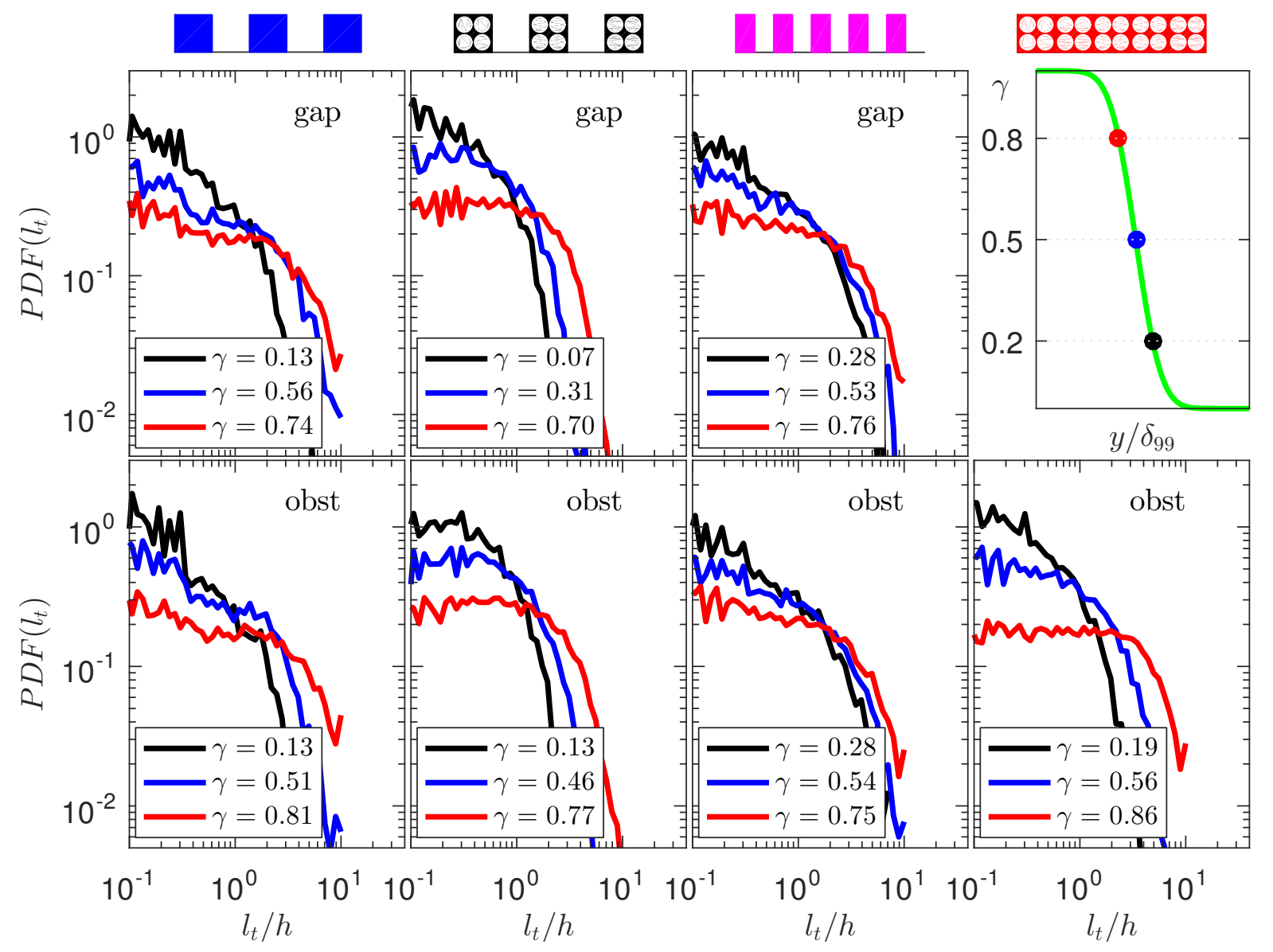

Figure 8: Probability density function $(P D F)$ of the length of the turbulent segments contained between two non-turbulent events (c.f. figure 2). Upper-right plot represents a schematic of the intermittency profile highlighting the approximate position of the three wall-normal locations (high-, medium- and low-intermittency levels) where the data is sampled.

considered. It is clear that the boundary-layer thickness has to be closely related to the trips' drag coefficient at this streamwise location (i.e. close to the trips, before the streamwise integral of the skin friction becomes large), which in turn is strongly dependent on the blockage ratio (e.g. Castro, 1971). This is in agreement with the fact that the holes trip presents a significantly smaller boundary-layer thickness since its blockage ratio is half that of the other trips. As alluded to previously the second contribution to the boundary-layer thickness is the streamwise integral of the friction coefficient. The fact that the fence trip presents smaller $\delta_{99}$ and $\theta$ than the base and AR2 trips may therefore be related to the smaller friction coefficient (i.e. larger $U_{\infty}^{+}$) shown in figure 3. To a lesser extent, this difference may also be observed for the base trip presenting a larger $\delta_{99}$ and larger friction coefficient than the AR2 case.

Naturally, a less disrupted boundary layer should more closely resemble a canonical TBL. On the other hand, a smaller degree of disruption also entails a thinner TBL which may be detrimental for certain engineering applications. We must therefore find a compromise between the degree of disruption of the turbulent structure and a sufficient increase of the TBL thickness. To do so it is thus of primary importance to know how different geometrical parameters affect the boundary layer behaviour as will be discussed below.

\section{1 base $\rightarrow$ holes}

In this instance the parameter being changed is the total blockage, $\sigma$, which is reduced from $50 \%$ to $25 \%$ by introducing perforations to the trips. The effect of doing this is to weaken the outer peak of turbulence intensity and making the velocity fluctuations more inhomogeneous/"less two-dimensional". Of all the trips the holes trip displays the most stark separation of scales between the inner peak and the outer peak. The inner peak is located almost perfectly at the expected length scale of $\lambda^{+}=1,000$ and is not as broad as for the other trips. In particular, there appears to be less influence from the fluctuations produced by the tip vortices, of larger $\lambda^{+}$, that generate the outer peak. This leads to a narrower intermittent region, i.e. constraining the outer disturbances from approaching the near-wall region, and the length of the turbulent segments appears to be more uniform than for the base flow, indicating a more regular TNTI position. All of these phenomena are 
synonymous with the flow becoming more wall-driven as described by Rodríguez-López et al. (2016b).

\section{2 base $\rightarrow A R \mathscr{Q}$}

In this instance the parameter being changed is the aspect ratio, $R$, which is increased from 1 to 2 . There is an increase in the total amount of turbulent kinetic energy, readily illustrated in figures 4 and 5 , however the spectra both look similar to one another (if they were to be normalised by $u_{r m s}^{\prime 2}$; not shown for brevity). The flow downstream also becomes more homogeneous as $R$ is increased from 1 to 2 . It is thus difficult to conclude one way or another whether the increase in $R$ pushes the flow towards either the wall-driven or wake-driven mechanism relative to the base trips. However, it can be seen that the $P D F$ of the length of the turbulent segments falls away less rapidly for the higher $A$ trips $(A R 2)$, suggesting at a slightly more irregular TNTI, which was previously linked to the wake-driven mechanism.

For this flow, it is reasonable to conjecture that very close to the obstacles the shear layer formed between their wakes and the free stream is produced by eddies dominated by spanwise vorticity which have been generated by fluid passing over the tips of the tripping obstacles. Contrastingly, the obstacles' wakes would be mainly populated by eddies with a wall-normal axis generated by the fluid passing between the trips, similar to the classical vortex shedding mechanism of a cylinder. This would imply that the shear-layer thickness scales with the typical lateral dimension of the trips $W$, and it is located at a wall-normal location scaling with $h$. Consequently a smaller outer-inner interaction would be expected for trips with a larger aspect ratio $h / W$ just because these eddies are located further from the wall relative to their size. Despite this paradigm not considering the streamwise growth of the shear layer, it supports the current results and suggests that the tested aspect ratios may indeed be insufficiently different so as to observe any significant difference between them. In fact, note that the cylinders employed by Rodríguez-López et al. (2016a) had aspect ratio $R=7.3$ which is much larger than the value of 2 tested here, and showed clear signs of thickening the TBL through the wall-driven mechanism.

\section{3 base $\rightarrow$ fence}

It is not straightforward to isolate a single parameter being changed in this instance. The wall blockage, $\sigma_{w}$ changes from $50 \%$ to $100 \%$ but the aspect ratio is undefined for the fence trip (whilst it is unity for the base trip). There is an increase in velocity fluctuation intensity with the introduction of the perforations with the fence trip generating the highest levels of turbulent fluctuations of any of the trips. de Bray (1957) showed that, generally, plates with a similar overall blockage $\sigma$ but with larger perforation holes would produce a lower velocity fluctuation intensity than plates with smaller holes. If one considers the gaps between the repeating obstacles of the base trip as "perforations", which are evidently bigger than the perforation holes of the fence trip, then this finding is in agreement with that for plates in a uniform free stream. Uniquely, due to the $100 \%$ wall blockage, the length scale associate to the inner peak of turbulent kinetic energy has been shifted to a smaller length scale, which appears to have reduced the skin friction (at this measurement station). However, the length scale of the outer peak is also shifted to a smaller value in comparison to the base trip meaning that there isn't a distinct separation of sacles between the inner and outer peak (as there is not for the base trip too). However, there is a substantial difference in the behaviour of the intermittent region. The perforation reduces the strength of the recirculation downstream of the trip thereby weakening the large-sale motions of the TNTI and leading to a thinner intermittent region with more regular turbulent segments. Whilst the flow shows many properties of the wake-driven mechanism, in particular the lack of separation of scales between the inner and outer peaks of turbulent kinetic energy, the evidence suggests that the influence of the wake may be weaker for the fence trip than the base trip.

\section{Conclusions}

Single-point hot-wire anemometry has been performed in the near field (15.6 trip heights) downstream of a series of spanwise-repeating obstacles to generate an artificially thick turbulent boundary layer. The trips were specifically designed to test three parameters that Rodríguez-López et al. (2016b) postulated would be significant in determining the nature of the adaptation region downstream of the trips which eventually transitions to a canonical, flat wall turbulent boundary layer. These were the aspect ratio of the trips, $R$, the frontal blockage, $\sigma$ and the blockage at the wall, $\sigma_{w}$. Attention was paid to two particular aspects of the flow, namely the onedimensional energy spectrum of the streamwise velocity fluctuations and the behaviour of the intermittent region of the outer part of the flow, which defines the wall-normal extent of the location of the turbulent/non-turbulent interface (TNTI).

The presence, or not, of porosity appears to be by far the biggest factor in determining the nature of the intermittent region. This is attributed to the fact that the bleed through of fluid from the upstream face of the 
trip re-energises the mean recirculation, thereby weakening the shedding of separated vortices. The two trips with porosity, holes and fence, had noticeably weaker shear layers between the turbulent and non-turbulent flow, resulting in narrower intermittent regions (with a steeper gradient of the intermittency function $\mathrm{d} \gamma / \mathrm{d} y$ ) and a more regular TNTI. Contrastingly, aspect ratio seemed to play little role in affecting the nature of the intermittent region over the range of $\mathbb{R}$ tested, with both solid trips of $\mathbb{R}=1$ (base) and $R=2$ (AR2) exhibiting similar behaviour.

The inner wall motions, characterised by the peak in energy content at $y^{+} \approx 15$ and $\lambda^{+} \approx 1,000$, were largely left undisturbed by all the trips that had a wall blockage of $50 \%$. However, this spectral peak was broadened by the solid trips (with $\sigma=0.5$ ) with contributions from larger length scales similar to those present in the outer peak, which is associated to the tip vortices by Rodríguez-López et al. (2016a). There was a clearer separation of scales, between the inner and outer peaks, for the lower blockage $(\sigma=0.25)$ holes trip. The inner motions were disturbed by the trip with $100 \%$ wall blockage, with a shift to a smaller $\lambda^{+}$and reduced wall shear stress (at this measurement location) evident for the fence trip. However, as stated, the effects of porosity are thought to dominate those of wall blockage and hence a better test case for examining the effects of $\sigma_{w}$ are the triangular sawtooth trips examined in Rodríguez-López et al. (2016a,b) which were associated with the wake-driven mechanism. Whilst it is difficult to decouple the effects of wall blockage from aspect ratio for these sawtooth trips we conclude from this study that $R$ had little effect on the flow downstream of the $A R 2$ trip relative to the base trip, with the exception of a higher turbulence intensity. This may also be attributed to the "perforations" (gaps) between the obstacles appearing to be smaller at the higher $R$ which has been previously shown to lead to higher turbulence intensities for flat plates of comparable blockage (de Bray, 1957).

As far as choosing the "best" of the trips tested in the present study are concerned the holes trips disrupted the inner peak the least (relative to a natural turbulent boundary layer) and produced a thinner intermittent region (i.e. a less tortuous TNTI). Both of these factors are synonymous with the wall-driven mechanism which was shown to produce canonical turbulent boundary layer properties earlier, after a shorter transition region in Rodríguez-López et al. (2016a). However, one must also think of the purpose of a boundary-layer tripping device since its primary purpose is to thicken a boundary layer (whilst retaining canonical properties). This will depend on the drag of the trips and hence will be a strong function of total blockage so the holes trips will produce a thinner boundary layer than the others. A compromise must therefore be sought between the amount of thickening and the available streamwise fetch to produce canonical turbulent boundary layer properties when choosing a suitable trip which will, in general, be applications specific.

OB and ERL would like to acknowledge the financial support given by European Union FP7 Marie Curie MULTISOLVE project (Grant Agreement No. 317269).

\section{References}

S.C.C. Bailey, M. Vallikivi, M. Hultmark, and A.J. Smits. Estimating the value of von Kármán's constant in turbulent pipe flow. Journal of Fluid Mechanics, 749:79-98, 2014.

D.K. Bisset, J.C.R. Hunt, and M.M. Rogers. The turbulent/non-turbulent interface bounding a far wake. Journal of Fluid Mechanics, 451:383-410, 2002.

I.P. Castro. Wake characteristics of two-dimensional perforated plates normal to an air-stream. Journal of Fluid Mechanics, 46(3):599-609, 1971.

K.A. Chauhan, P.A. Monkewitz, and H.M. Nagib. Criteria for assessing experiments in zero pressure gradient boundary layers. Fluid Dynamics Research, 41(2):021404, 2009.

K.A. Chauhan, J. Philip, C.M. de Silva, N. Hutchins, and I. Marusic. The turbulent/non-turbulent interface and entrainment in a boundary layer. Journal of Fluid Mechanics, 742:119-151, 2014.

D. Coles. The law of the wake in the turbulent boundary layer. Journal of Fluid Mechanics, 1(2):191-226, 1956.

G. Comte-Bellot. Hot-wire anemometry. Annual Review of Fluid Mechanics, 8:209-231, 1976.

J. Counihan. An improved method of simulating an atmospheric boundary layer in a wind tunnel. Atmospheric Environment (1967), 3(2):197-214, 1969.

M.J. Davidson, W.H. Snyder, R.E. Lawson, and J.C.R. Hunt. Wind tunnel simulations of plume dispersion through groups of obstacles. Atmospheric Environment, 30(22):3715-3731, 1996.

B.G. de Bray. Low speed wind tunnel tests on perforated square flat plates normal to the airstream: Drag and velocity fluctuation measurements. Technical Report CP 323, Aeronautical Research Council, 1957.

E. Dogan, R.E. Hanson, and B. Ganapathisubramani. Interactions of large-scale free-stream turbulence with turbulent boundary layers. Journal of Fluid Mechanics, 802:79-107, 2016.

L.B. Esteban, E. Dogan, E. Rodríguez-López, and B. Ganapathisubramani. Skin-friction measurements in a turbulent boundary layer under the influence of free-stream turbulence. Experiments in Fluids, 58(9):115, 2017. 
J.C.R. Hunt and H. Fernholz. Wind-tunnel simulation of the atmospheric boundary layer: a report on euromech 50. Journal of Fluid Mechanics, 70(3):543-559, 1975.

N. Hutchins and I. Marusic. Large-scale influences in near-wall turbulence. Philosophical Transactions of the Royal Society of London A: Mathematical, Physical and Engineering Sciences, 365:647-664, 2007.

P.S. Klebanoff. Characteristics of turbulence in a boundary layer with zero pressure gradient. Technical Report 1247, NACA, 1955.

P.S. Klebanoff and Z.W. Diehl. Some features of artificially thickened fully developed turbulent boundary layers with zero pressure gradient. Technical Report 1110, NACA, 1952.

J.C. Klewicki. Reynolds number dependence, scaling, and dynamics of turbulent boundary layers. Journal of Fluids Engineering, 132(9):094001-094001-48, 2010. 10.1115/1.4002167.

S.J. Kline, W.C. Reynolds, F.A. Schraub, and P.W. Runstadler. The structure of turbulent boundary layers. Journal of Fluid Mechanics, 30(4):741-773, 1967.

V.I. Kornilov and A.V. Boiko. Wind-tunnel simulation of thick turbulent boundary layer. Thermophysics and Aeromechanics, 19(2):247-258, 2012.

V.I. Kornilov and A.V. Boiko. Simulation of a thick turbulent boundary layer via a rod grid. Thermophysics and Aeromechanics, 20(3):289-302, 2013.

I. Marusic, B.J. McKeon, P.A. Monkewitz, H.M. Nagib, A.J. Smits, and K.R Sreenivasan. Wall-bounded turbulent flows at high Reynolds numbers: Recent advances and key issues. Physics of Fluids, 22(065103): $1-24,2010$.

I. Marusic, J.P. Monty, M. Hultmark, and A.J. Smits. On the logarithmic region in wall turbulence. Journal of Fluid Mechanics, 716, 2013.

A. J. Musker. Explicit expression for the smooth wall velocity distribution in a turbulent boundary layer. $A I A A$ Journal, 17(6):655-657, 1979.

H.M. Nagib and K.A. Chauhan. Variations of von Kármán coefficient in canonical flows. Physics of Fluids, 20 (10):101518:1-10, 2008.

T.B. Nickels, I. Marusic, S. Hafez, N. Hutchins, and M.S. Chong. Some predictions of the attached eddy model for a high reynolds number boundary layer. Philosophical Transactions of the Royal Society A: Mathematical, Physical and Engineering Sciences, 365(1852):807-822, 2007.

E. Rodríguez-López, P.J.K. Bruce, and O.R.H. Buxton. A robust post-processing method to determine skin friction in turbulent boundary layers from the velocity profile. Experiments in Fluids, 56(4):1-16, 2015.

E. Rodríguez-López, P.J.K. Bruce, and O.R.H. Buxton. On the formation mechanisms of artificially generated high Reynolds number turbulent boundary layers. Boundary-Layer Meteorology, 160(2):201-224, 2016a.

E. Rodríguez-López, P.J.K. Bruce, and O.R.H. Buxton. Near field development of artificially generated high Reynolds number turbulent boundary layers. Physical Review Fluids, 1(7):074401:1-22, $2016 \mathrm{~b}$.

E. Rodríguez-López, P.J.K. Bruce, and O.R.H. Buxton. Flow characteristics and scaling past highly porous wall-mounted fences. Physics of Fluids, 29(7):075106:1-20, 2017a.

Eduardo Rodríguez-López, Paul J. K. Bruce, and Oliver R. H. Buxton. Experimental measurement of wall shear stress in strongly disrupted flows. Journal of Turbulence, 18(3):271-290, $2017 \mathrm{~b}$.

C. Sanmiguel Vila, R. Vinuesa, S. Discetti, A. Ianiro, P. Schlatter, and R. Örlü. On the identification of well-behaved turbulent boundary layers. Journal of Fluid Mechanics, 822:109-138, 2017.

K. Steiros, P.J.K. Bruce, O.R.H. Buxton, and J.C. Vassilicos. Effect of blade modifications on the torque and flow field of radial impellers in stirred tanks. Physical Review Fluids, In press, 2017.

G.I. Taylor. The spectrum of turbulence. Proceedings of the Royal Society of London A: Mathematical, Physical and Engineering Sciences, 164(919):476-490, 1938. 